\title{
Numerical Estimations of Carrier Generation-Recombination Processes and the Photon Recycling Effect in HgCdTe Heterostructure Photodiodes
}

\author{
K. JÓŹWIKOWSKI ${ }^{1}$ M. KOPYTKO, ${ }^{1,2}$ and A. ROGALSKI ${ }^{1}$ \\ 1.--Institute of Applied Physics, Military University of Technology, 2 Kaliskiego St., 00-908 \\ Warsaw, Poland. 2.—e-mail: mkopytko@wat.edu.pl
}

An enhanced computer program has been applied to explain in detail the photon recycling effect which drastically limits the influence of radiative recombination on the performance of $p$-on- $n \mathrm{HgCdTe}$ heterostructure photodiodes. The computer program is based on a solution of the carrier transport equations, as well as the photon transport equations for semiconductor heterostructures. We distinguish photons in two energy ranges according to $p^{+}$ and $n$ region with unequal band gaps. As a result, both the distribution of thermal carrier generation and recombination rates and spatial photon density distribution in photodiode structures have been obtained. The general conclusion, similar to our earlier work concerning $3-\mu \mathrm{m} n$-on- $p \mathrm{HgCdTe}$ heterostructure photodiodes, confirms the previous assertion by Humphreys that radiative recombination does not limit $\mathrm{HgCdTe}$ photodiode performance.

Key words: Photon recycling, generation-recombination processes, HgCdTe photodiodes

\section{INTRODUCTION}

Taking into account the volume carrier generation and recombination processes, three fundamental mechanisms of thermal generation and recombination should be considered in $\mathrm{HgCdTe}$ ternary alloy: radiative transitions, and Auger 1 and Auger 7 recombination. Additionally, the nonfundamental Shockley-Read-Hall (SRH) recombination mechanism is associated with trap states (mercury vacancies) and dislocations. ${ }^{1}$ Since the $1970 \mathrm{~s}$, all these mechanisms have been the subject of extensive experimental and theoretical research. ${ }^{2}$ While the influence of SRH mechanisms can be limited by reducing the density of trap states in the technological processes, the radiative and Auger mechanisms represent a fundamental limit on the rate of thermal generation (or recombination) of charge carriers. However, if the charge carrier density can be held below its equilibrium value, an improved signal-to-noise ratio will result in a

(Received October 11, 2011; accepted March 24, 2012;

published online April 18, 2012) suitable device structure. ${ }^{3,4}$ Auger suppression as well as low radiative recombination and $\mathrm{SRH}$ generation are required to achieve a backgroundlimited infrared performance (BLIP) response near room temperature for both near-infrared low-lightlevel and thermal imaging applications.

Humphreys asserts that traditional radiative recombination is unlikely to limit performance in properly designed photodiodes. ${ }^{5}$ The phenomenon known as photon recycling (PR), or reabsorption within semiconductors of photons generated by minority carrier recombination, increases the minority carrier lifetime above that estimated by the traditional theory of van Roosbroeck and Shockley (VRS). ${ }^{6}$ Due to reabsorption, the radiative lifetime is greatly extended and dependent on the semiconductor geometry, the absorption coefficient, and the refractive index. It was estimated that the lifetime increases with increasing refraction index, absorption coefficient, and sample thickness. ${ }^{7}$

Despite the above predictions, in the majority of papers the traditional VRS theory is used to estimate $\mathrm{HgCdTe}$ detector performance. The main reason for this is that formal modeling of the $\mathrm{PR}$ 
effect requires inclusion of additional terms in the standard set of carrier and photon transport equations, and researchers usually tend to neglect its influence because of the lack of available tools for easy evaluation of this phenomenon in their practical devices. The calculations are usually based on the use of commercially available programs for semiconductor device simulation, which initially do not have the potential for PR modeling.

However, when reabsorption is included, there are two energy interchange processes which need to be considered in any given volume of a semiconductor element: that between carriers and photons within the element, and that between photons within the element and the cavity surrounding it. Therefore, to include PR in detailed modeling of devices, the photon transport equation should be included in the set of carrier transport equations. ${ }^{8-10}$ For photons with energy less than the band gap energy $\left(h v<E_{\mathrm{g}}\right)$, absorption, emission, and scattering are weak and the wave equation describes very thoroughly the overall photon propagation. Mathematically, the equations of propagation are hyperbolic partial differential equations, and their numerical solution is relatively easy and stable. To analyze the PR effect, only photons with energy equal to or higher than the band gap, $h v \geq E_{\mathrm{g}}$, are essential. For these photons, however, the probability that a photon propagates in a straight line unhindered is very low; radiative transport is dominated by many scattering, absorption, and reabsorption events. The emitted photons, in turn, are quickly reabsorbed. The net emission (or absorption) is then the small difference between two large terms. More generally, in semiconductor devices, radiation with energy $h v \geq E_{\mathrm{g}}$ tends to be nearly isotropic and, eventually, close to local thermal equilibrium.

This work is a continuation of our earlier work ${ }^{10}$ in which we presented results of calculations performed for $n$-on- $p$ homojunction HgCdTe photodiodes. The analysis of carrier generation-recombination $(\mathrm{g}-\mathrm{r})$ processes and the photon recycling effect was based on numerical solution of the carrier transport equations including a simplified version of the transport equations for selected photons with energy greater than the energy gap. In heterostructure devices, the problem is further complicated by the spatial variation of the energy gap. Photons generated and reabsorbed in an area with narrower energy gap are not reabsorbed in areas with a wider energy band gap. Therefore, it becomes necessary to analyze photons in different frequency ranges separately and formulate their respective balance equations.

The principal purpose of this paper is to present numerical methods for solving the transport equations for the electric charge carriers and photons connected with carriers in semiconductor heterostructures. We have developed a previous model that can be applied for heterostructure photodiodes.
Despite the fact that the presented results have been obtained for simple heterostructure photodiodes with only two areas with different widths of energy gap, we also show how to use the method for more sophisticated structures.

The results of the calculations show that photons emitted by radiative recombination events and then reabsorbed in the radiative generation process cause a considerable reduction of the carrier radiative generation (or recombination) rates. Our results once again confirm Humphreys' assertion ${ }^{5,7}$ that the influence of radiative recombination on $\mathrm{HgCdTe}$ photodiode performance is greatly overestimated.

\section{ANALYSIS METHOD}

Humphreys' calculations of the PR effect apply to materials with an equal energy gap. ${ }^{5,7}$ In further considerations, we assume a procedure described by Humphreys to include the PR effect for heterostructures. If there are areas with a different energy gap in a semiconductor, we should take into account photons with energies corresponding to the appropriate range $E^{i}<h v \leq E^{i}+\Delta E^{i}$ from $i=1$ to $i=m$, where $E^{1}=E_{\mathrm{g}}^{\min }$ and $E^{n}+\Delta E^{n}=\infty$. Here, $E_{\mathrm{g}}^{\min }$ denotes the lowest energy gap in the heterostructure. Then, the optical generation rate per unit volume can be expressed as

$$
G_{\mathrm{RAD}}=\sum_{i=1}^{m} G_{\mathrm{RAD}}^{i}
$$

where $^{6}$

$$
G_{\mathrm{RAD}}^{i}=\int_{E^{i}}^{E^{i}+\Delta E^{i}} \frac{c q(v) \alpha(v)}{\eta(v)} \mathrm{d} v,
$$

where $v$ is the frequency, $\eta(v)$ is the refractive index, $c$ is the velocity of light, $\alpha(v)$ is the absorption coefficient, and $q$ is the photon density as determined by Planck's law.

In accordance with Humphreys, the quantities in Eq. 2 can be expressed by appropriate averages

$$
\begin{gathered}
q_{0}^{i}=\int_{E^{i}}^{E^{i}+\Delta E^{i}} q(v) \mathrm{d} v, \\
\frac{\alpha^{i}}{\eta^{i}}=\frac{1}{q_{0}^{i}} \int_{E^{i}}^{E^{i}+\Delta E^{i}} \frac{q(v) \alpha(v)}{\eta(v)} \mathrm{d} v .
\end{gathered}
$$
by

The generation rate per unit volume is then given

$$
G_{\mathrm{RAD}}=\sum_{i=1}^{m} G_{\mathrm{RAD}}^{i}=\sum_{i=1}^{m} q_{0}^{i} \alpha^{i} \frac{c}{\eta^{i}} .
$$


The radiative recombination rate per unit volume is proportional to the product of the electron and hole concentrations: $R_{\mathrm{RAD}}=k n p$ (see, e.g., Ref. 11). In equilibrium, following VRS we have ${ }^{6} R_{\mathrm{RAD}}=k n_{0} p_{0}=$ $G_{\mathrm{RAD}}$, and then $k=R_{\mathrm{RAD}} / n_{0} p_{0}$, and the net radiative recombination rate is

$$
R_{\mathrm{RAD}}=\sum_{i=1}^{m} R_{\mathrm{RAD}}^{i}
$$

where

$$
R_{\mathrm{RAD}}^{i}=G_{\mathrm{RAD}}^{i} \frac{n p}{n_{0} p_{0}}=q_{0}^{i} \alpha^{i} \frac{c}{\eta^{i}} \frac{n p}{n_{0} p_{0}} .
$$

The classical VRS equation for the net radiative recombination rate is 6

$$
R_{\mathrm{RAD}}-G_{\mathrm{RAD}}=\sum_{i=1}^{m} q_{0}^{i} \alpha^{i} \frac{c}{\eta^{i}} \frac{n p}{n_{0} p_{0}}-\sum_{i=1}^{m} q_{0}^{i} \alpha^{i} \frac{c}{\eta^{i}} .
$$

In nonequilibrium conditions, the photon density $q^{i} \neq q_{0}^{i}$ and Eq. 6 is modified to

$$
R_{\mathrm{RAD}}^{\mathrm{PR}}-G_{\mathrm{RAD}}^{\mathrm{PR}}=\sum_{i=1}^{m} q_{0}^{i} \alpha^{i} \frac{c}{\eta^{i}} \frac{n p}{n_{0} p_{0}}-\sum_{i=1}^{m} q^{i} \alpha^{i} \frac{c}{\eta^{i}},
$$

due to change in the radiative generation rate. The first term of Eq. 9 determines the photon generation rate due to radiative recombination, and the second term defines the photon annihilation rate caused by absorption. If, in any unit of semiconductor volume $\mathrm{d} V$, there is a change in the photon density, then depending on the sign of Eq. 9 this element emits or absorbs part of the photons.

The photon balance in a given element of volume can be written assuming a simplified version of the transport equations. The transport equation for photons of density $q^{i}$ with energy $E^{i}<h v \leq E^{i}+\Delta E^{i}$ is expressed as

$$
\frac{\partial q^{i}}{\partial t}=G_{\mathrm{RAD}}^{i} \frac{n p}{n_{0} p_{0}}-q^{i} \alpha^{i} \frac{c}{\eta^{i}}-\nabla j_{q}^{i},
$$

where $i=1,2, \ldots, m \cdot j_{q}^{i}=q^{i} c / 4 \eta^{i 3}$ is the photon current density. Here, a source of additional photon generation is carrier radiative recombination, and the source of their annihilation is electron-hole pair generation events. The final term in Eq. 10 represents the divergence of the photon current.

In the next step we include these $m$ equations (10) into the set of four charge carrier transport equations comprising the continuity equations for electrons and holes, Poisson's equation, and the thermal conductivity equation. These equations and the numerical method for their solution were described in our earlier paper. ${ }^{10}$

\section{RESULTS OF CALCULATIONS}

In this paper we consider the $p^{+}-n$ HgCdTe heterostructure photodiode shown in Fig. 1a. This
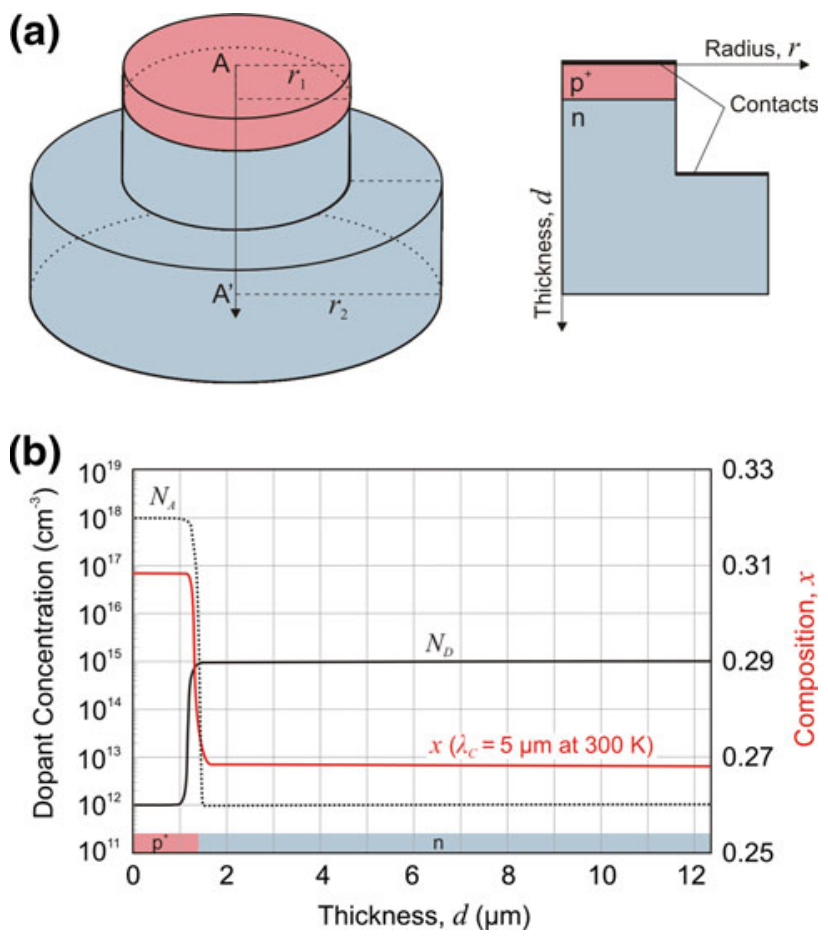

Fig. 1. Cross-section of the $p$-on- $n \mathrm{HgCdTe}$ mesa photodiode structure (a) and the doping and composition profiles (b). The line $\mathrm{AA}^{\prime}(d)$ indicates the direction of calculated spatial distributions of different parameters. Device geometry: $r_{1}=34 \mu \mathrm{m}, r_{2}=58 \mu \mathrm{m}$, and $d=12.35 \mu \mathrm{m}$.

photodiode structure is mainly produced by liquidphase epitaxy (LPE), molecular beam epitaxy (MBE), or metalorganic chemical vapor deposition (MOCVD) on a lattice-matched CdZnTe substrate. The doping profiles of the analyzed photodiode are shown in Fig. 1b. An absorber layer approximately $11 \mu \mathrm{m}$ thick is $n$-type doped at $1 \times 10^{15} \mathrm{~cm}^{-3}$. The $n$-type $\mathrm{Hg}_{1-x} \mathrm{Cd}_{x} \mathrm{Te}$ carrier concentration is easy to control in the $10^{15} \mathrm{~cm}^{-3}$ range using extrinsic doping, usually by indium or iodine. The composition of an absorber layer has been determined for photodiodes with cutoff wavelengths equal to $5 \mu \mathrm{m}$ and $11 \mu \mathrm{m}$ operating at $80 \mathrm{~K}$ and $300 \mathrm{~K}$. The widerband-gap $p$-type capping $\mathrm{Hg}_{1-y} \mathrm{Cd}_{y} \mathrm{Te}(y \approx x+0.04)$ is about $1 \mu \mathrm{m}$ thick and is not intentionally doped. We choose the following composition $x$ in the $n$-type region:

- $\lambda_{\mathrm{c}}=5 \mu \mathrm{m}$ at $T=80 \mathrm{~K}, x=0.3$; at $T=300 \mathrm{~K}$, $x=0.27$

- $\lambda_{\mathrm{c}}=11 \mu \mathrm{m}$ at $T=80 \mathrm{~K}, x=0.22$; at $T=300 \mathrm{~K}$, $x=0.17$

Figure $1 \mathrm{~b}$ shows an example composition profile for a $p$-on- $n$ HgCdTe photodiode with cutoff wavelength of $5 \mu \mathrm{m}$ operating at $300 \mathrm{~K}$.

The numerical analysis has been realized assuming that the structure is illuminated by background flux. We have also assumed that the structure and its surrounding has the same temperature. The absorption coefficient is estimated 
(a)

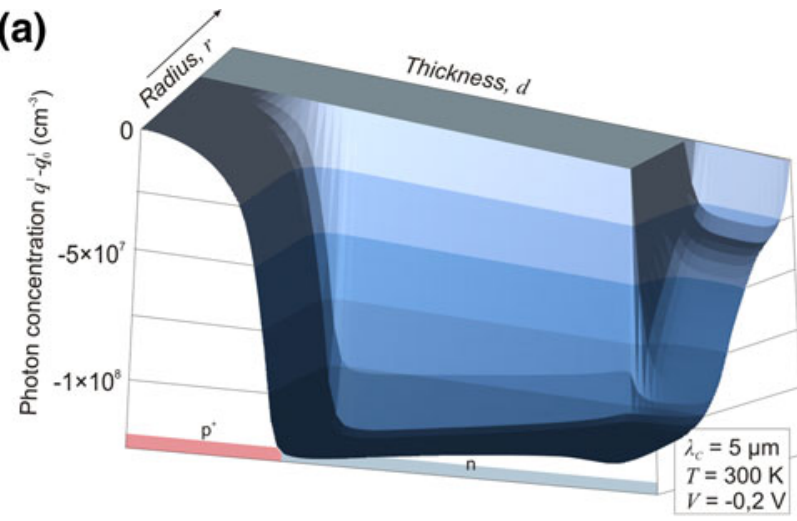

(c)

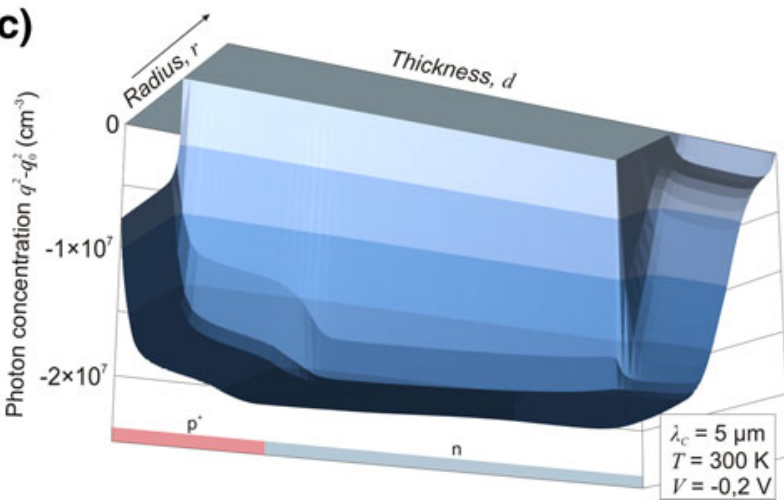

(b)

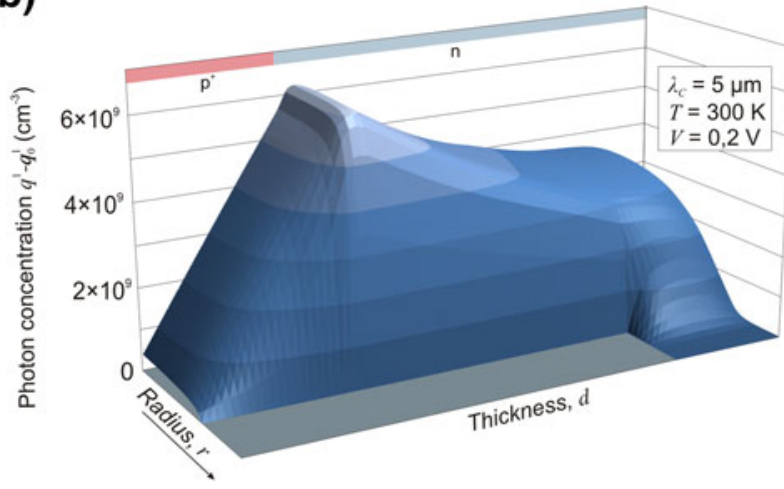

(d)

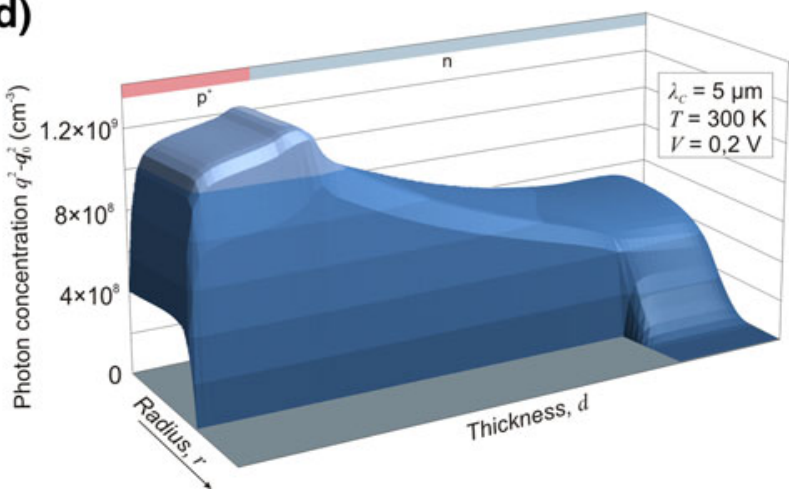

Fig. 2. Spatial distribution of changes of photon densities $\left(q^{i}-q_{0}^{i}\right)$ in a reverse (a, c) and forward (b, d) biased 5- $\mu \mathrm{m} p$-on- $n$ HgCdTe photodiode operating at temperature of $300 \mathrm{~K}$ for photons $q^{1}$ with energy $E_{\mathrm{g}}^{n} \leq h v<E_{\mathrm{g}}^{p^{+}}(\mathrm{a}, \mathrm{b})$ and photons $q^{2}$ with energy $h v \geq E_{\mathrm{g}}^{p^{+}}$(c, d).

according to Anderson's model, ${ }^{12}$ and the refractive index was taken from Ref. 13. The remaining parameters are taken from Ref. 14. We assume a uniform mercury vacancy density, corresponding to SRH trap centers, of $1 \times 10^{12} \mathrm{~cm}^{-3}$ in all considered heterostructure devices.

The method of calculation of the carrier transport equations presented in Ref. 10 can be used for semiconductor heterostructures. Since different regions of the heterostructure are characterized by different energy gaps, due to the various refractive indexes and cutoff wavelengths, it is necessary to distinguish photons in different energy ranges. We have distinguished two groups of photons, one with density $q^{1}$ and energy $E_{\mathrm{g}}^{n} \leq h v<E_{\mathrm{g}}^{p^{+}}$and the second group of photons with density $q^{2}$ and energy $h v \geq E_{\mathrm{g}}^{p^{+}} . E_{\mathrm{g}}^{n}$ denotes the energy of the $n$-type absorber region, and $E_{\mathrm{g}}^{p^{+}}$denotes the energy of the $p^{+}$region.

By introducing two additional equations (Eq. 10) for $i=1,2$ into the set of charge transport equations presented in Ref. 10, we can take into account the PR effect for photons in two energy ranges and thereby effectively estimate the carrier radiative recombination and generation rates. Analyzing Eq. 10 for $i=1,2$ it can be noted that both $q^{1}$ as well as $q^{2}$ photons are involved in the radiative recombination or generation process; however, photons $q^{1}$ are of importance only in the absorber area of the analyzed photodiodes. The reverse bias causes depletion of the charge carriers (extraction effect), being especially strong in the region of the $p-n$ junction. This is the reason for the reduction in the rate of carrier radiative recombination, thus leading to decreased photon densities, both $q^{1}$ as well as $q^{2}$. This phenomenon is well illustrated by Fig. 2a, c, in which the spatial distributions of the changes of the photon densities in a reverse-biased photodiode with cutoff wavelength of $5 \mu \mathrm{m}$ operating at $300 \mathrm{~K}$ are presented. The largest reduction in the photon density is observed in the $p-n$ junction. Near the photodiode surface, changes of the photon density are smaller due to the photons inflowing from the environment (background). The presented results seem well suited to quantitative modeling of the nonequilibrium IR detectors by Ashley and Elliott ${ }^{15}$ that use carrier extraction under reverse bias to increase the Auger and radiative lifetimes and/or obtain negative luminescent devises. ${ }^{16-18}$ The forward bias leads to an increase in the charge carrier concentration, and thus to an increase in carrier radiative recombination, which is a source of additional photons. As a result, an increase in the photon density is observed. Figure $2 b$, $d$ shows the spatial distribution of the changes of photon densities in a forward-biased photodiode with cutoff wavelength of $5 \mu \mathrm{m}$ operating at $300 \mathrm{~K}$. In this case, changes of the photon density at the surface of the 
photodiode are also smaller due to the emission of photons by the diode to the environment. In this case, positive luminescence is observed. ${ }^{17}$

Although the reason for the changes in the photon density on biasing is easy to explain, analysis of the spatial distribution of the carrier radiative recombination (generation) rate is not so simple:

- If the carrier concentration is lower than the equilibrium one, the first term of Eq. 10 for $i=1$, 2 decreases; in this case, the number of photons capable of carrier generation decreases and as a consequence the second term may also decrease. So, the resulting net generation depends on the change in both values. The situation is further complicated by the fact that the total net generation rate is the result of summing the contribution of photons $q^{1}$ and $q^{2}$.

- However, if the carrier concentration is higher than the equilibrium one, the first term of Eq. 10 for $i=1,2$ increases, but also the second term increases due to the generation of additional photons $\left(q^{1}>q_{0}^{1}\right.$ and $\left.q^{2}>q_{0}^{2}\right)$. Similarly to the first case, the resulting net generation can increase or decrease depending on the size of the change in the two components.

- Besides the generation of photons in the semiconductor structure, Eq. 10 (for $i=1,2$ ) takes into account the impact of photon fluxes on their density.

The spatial distribution of the net carrier radiative recombination (generation) rates in mid-wavelength infrared (MWIR) $p$-on- $n$ HgCdTe photodiodes operating at $300 \mathrm{~K}$ and $80 \mathrm{~K}$ are presented in Figs. 3 and 4 , respectively. The diagrams are aligned along the line $\mathrm{AA}^{\prime}$ (Fig. 1). The results of calculations for the classical case (VRS theory) are simple to interpret. Reducing the carrier concentration in the reverse-biased photodiode reduces the rate of carrier radiative recombination, while the rate of carrier radiative generation, which does not depend (according to VRS theory) on the carrier concentration, does not change. Therefore, in a reversebiased photodiode, the net radiative generation prevails. The opposite situation applies in a forward-biased photodiode.

The net spatial distribution of the recombination (generation) rates with the PR effect thereby changes sign, so that regions in which net radiative recombination or generation prevails occur alternately. Let us consider this behavior in detail. For this goal, Eq. 10 can be expressed as

$$
\left(R_{\mathrm{RAD}}^{\mathrm{PR}}-G_{\mathrm{RAD}}^{\mathrm{PR}}\right)^{i}=q^{i} \alpha^{i} \frac{c}{\eta^{i}}\left(\frac{n p}{n_{0} p_{0}} \frac{q_{0}^{i}}{q^{i}}-1\right)=q^{i} \alpha^{i} \frac{c}{\eta^{i}}\left(\gamma^{i}-1\right),
$$

where

$$
\gamma^{i}=\frac{n p}{n_{0} p_{0}} \frac{q_{0}^{i}}{q^{i}}
$$
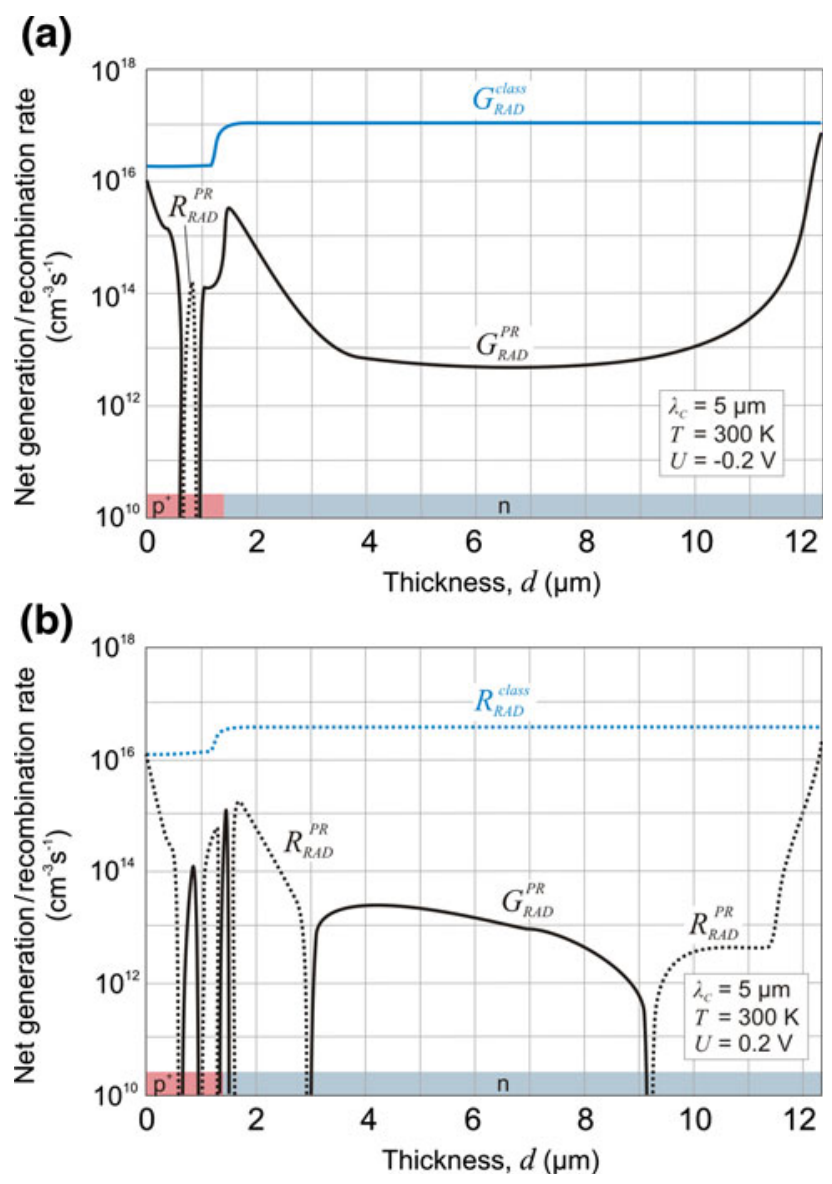

Fig. 3. Spatial distribution of the net recombination (generation) rates in a reverse (a) and forward (b) biased $p$-on- $n$ HgCdTe photodiode with $5-\mu \mathrm{m}$ cutoff wavelength operating at $300 \mathrm{~K}$. Bias voltage is $0.2 \mathrm{~V}$.

and $i=1,2$. In Fig. 5, the spatial distribution of the factors $\gamma^{i=1,2}$ are shown together with the net recombination and generation rates in a reversebiased $\mathrm{HgCdTe}$ photodiode with 5 - $\mu \mathrm{m}$ cutoff wavelength operating at $300 \mathrm{~K}$.

Analyzing Eq. 11 we notice that the sign of the net carrier radiative recombination and generation is connected both with changing the carrier concentration $\left(n p / n_{0} p_{0}\right)$ and with changing the photon density $\left(q^{i} / q_{0}^{i}\right)$. With the dependence (11), the result shows that, if $n p / n_{0} p_{0}$ is larger or smaller than $\left(q^{i} / q_{0}^{i}\right)$, the factor $\gamma^{i}$ is larger or smaller than 1 , respectively.

In a reverse-biased photodiode, the number of photons generated decreases (Fig. 2a). Then, $q_{0}^{i} / q^{i}>1$. However, simultaneously, carrier exclusion occurs inside the structure, especially strongly in the area of the $p-n$ junction and in the weakly doped $n$-type layer. Then, $n p / n_{0} p_{0}<1$. The carrier exclusion effect in the mentioned regions is stronger than the suppression of photons and the factor $\gamma^{i}$ is smaller than 1 , for photons with density $q^{1}$ as well as those with density $q^{2}$. The carrier exclusion accompanies the increased carrier radiative generation in 


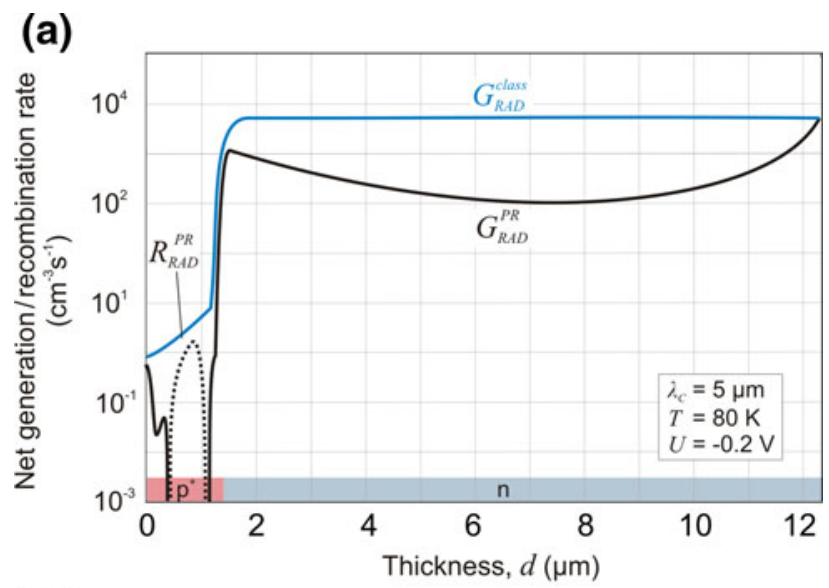

(b)

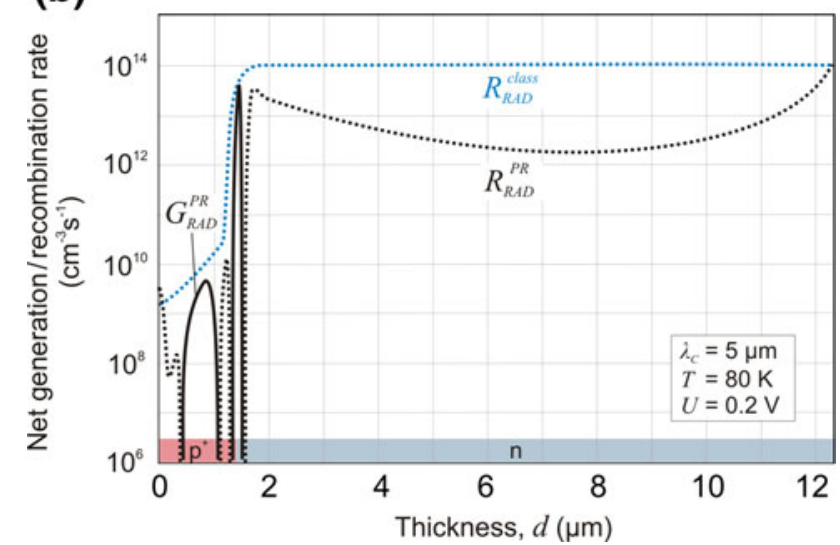

Fig. 4. Spatial distribution of the net recombination (generation) rates in a reverse (a) and forward (b) biased $p$-on- $n$ HgCdTe photodiode with $5-\mu \mathrm{m}$ cutoff wavelength operating at $80 \mathrm{~K}$. Bias voltage is $0.2 \mathrm{~V}$.

the $n$-type region and the region of the $p-n$ junction (Fig. 5a, b). In this case, these two opposed processes (carrier exclusion and photon suppression) result in the fact that, in the reverse-biased $\mathrm{HgCdTe}$ photodiode with cutoff wavelength of $5 \mu \mathrm{m}$ operating at $300 \mathrm{~K}$, the resultant net generation rate is almost four orders of magnitude lower than the value estimated from the VRS theory (Fig. 3a). A similar situation occurs for the photodiode operated at $80 \mathrm{~K}$; nevertheless, the PR effect is weaker.

Since photons $q^{1}$ with energy $E_{\mathrm{g}}^{n} \leq h v<E_{\mathrm{g}}^{p^{+}}$cannot be absorbed in the $p^{+}$region with wider energy band gap, only photons $q^{2}$ with energy $h v \geq E_{\mathrm{g}}^{p^{+}}$may be considered. In the highly doped $p^{+}$region, the carrier exclusion effect practically does not exist, so $n p \approx n_{0} p_{0}$. However, even slight suppression of photons (Fig. 2c) results in the fact that the factor $\gamma^{2}$ is larger than 1 (Fig. 5b), causing the increased radiative recombination. Therefore, this region (B) is the source of generated photons, which are reabsorbed in the neighboring regions $\mathrm{A}$ and $\mathrm{C}$, leading to the local dominance of the carrier generation rate over their radiative recombination in these regions. Moreover, the near-surface regions $\mathrm{A}$ and $\mathrm{E}$ are
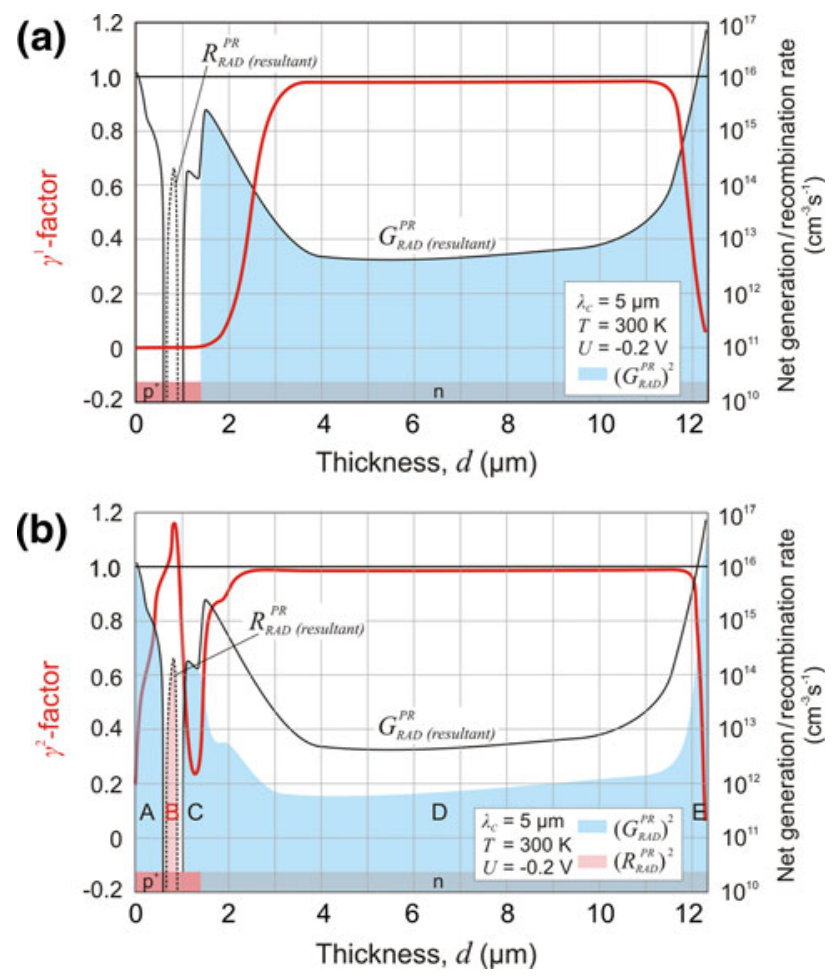

Fig. 5. Spatial distribution of the $\gamma$ factor and net recombination and generation rates for a reverse-biased 5- $\mu \mathrm{m} p$-on- $n \mathrm{HgCdTe}$ photodiode operating at temperature of $300 \mathrm{~K}$ for photons $q^{1}$ with energy $E_{\mathrm{g}}^{n} \leq h v<E_{\mathrm{g}}^{p^{+}}$(a) and photons $q^{2}$ with energy $h v \geq E_{\mathrm{g}}^{p^{+}}$(b).

"stabilized" by the constant photon flux inflowing from the environment (background). Thus, the net radiative recombination rate differs little from the prediction of Eq. 8.

Forward biasing results in a more complex situation (Figs. 3b, 4b). A very similar case has been explained in detail for the $n$-on- $p$ HgCdTe photodiode in our previous work. ${ }^{10}$ For heterostructure devices with areas with different energy gaps, it is necessary to analyze photons in different frequency ranges separately; nevertheless, only g-r processes occurring in the active area of photodiodes ( $n$-type absorber) determine their parameters, as clearly illustrated in Fig. 5a, b. The influence of photons with energy $E_{\mathrm{g}}^{n} \leq h v<E_{\mathrm{g}}^{p^{+}}$is much smaller than for photons with energy $h v \geq E_{\mathrm{g}}^{p^{+}}$.

The general conclusion is the following: The photon recycling effect leads to local oscillations of the net radiative generation rate, with maximal values several orders of magnitude lower than predicted by the RS theory. Only in small, near-surface regions (order of $1 \mu \mathrm{m}$ ) does the photon recycling effect have any importance due to the influence of background radiation. It appears that the suppression of the radiative recombination (generation) rate by the PR effect is observed at a moderate bias voltage.

Under the condition of a small excess carrier concentration, the classical radiative lifetime (using the VRS theory) can be calculated as 

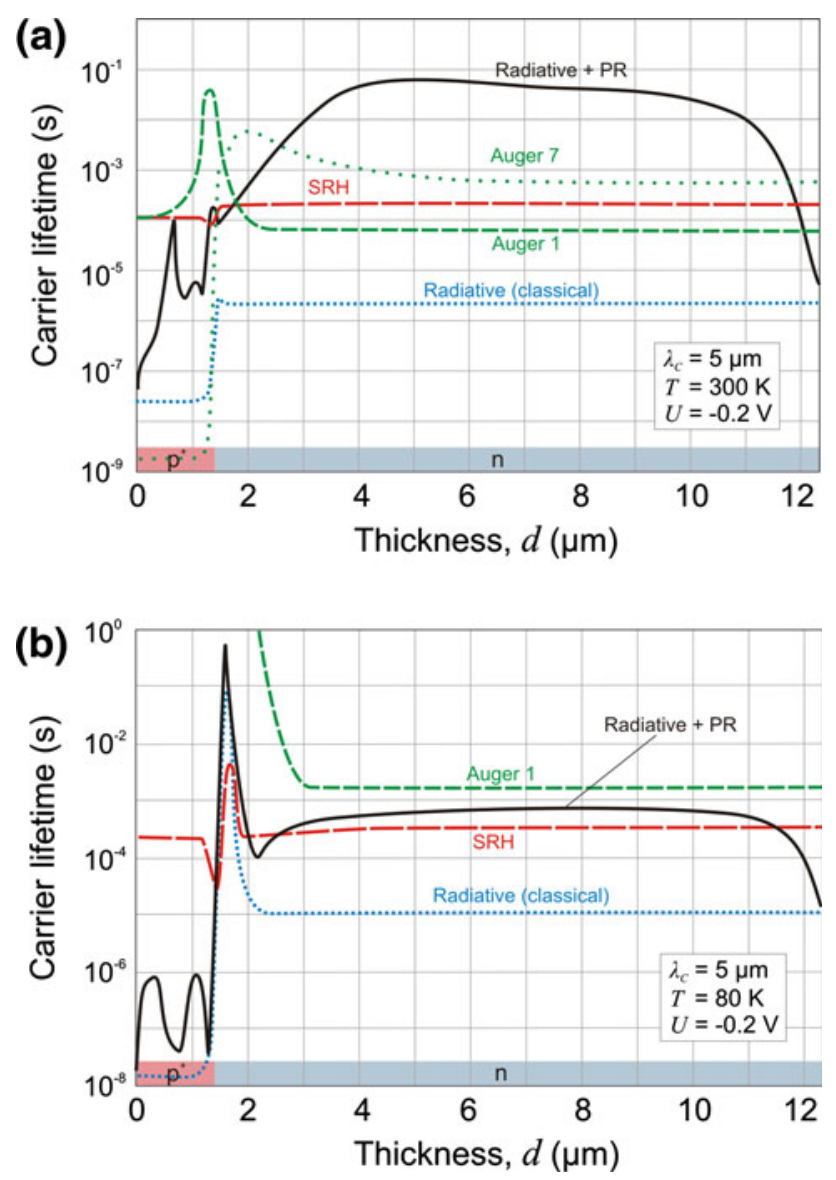

Fig. 6. Spatial distribution of carrier lifetime in a reverse-biased $5-\mu \mathrm{m}$ $p$-on- $n \mathrm{HgCdTe}$ photodiode operating at $300 \mathrm{~K}$ (a) and $80 \mathrm{~K}$ (b). Reverse bias voltage is $0.2 \mathrm{~V}$.

$$
\tau_{\mathrm{RAD}}^{\text {class }}=\frac{n-n_{0}}{R_{\mathrm{RAD}}-G_{\mathrm{RAD}}}=\frac{n_{0} p_{0}}{n_{0}+p_{0}}\left[\sum_{i=1}^{m} \frac{\eta^{i}}{q_{0}^{i} \alpha^{i} c}\right],
$$

and if the PR effect is included

$$
\tau_{\mathrm{RAD}}^{\mathrm{PR}}=\frac{n p-n_{0} p_{0}}{n_{0}+p_{0}}\left[\sum_{i=1}^{m} \frac{\eta^{i}}{q_{0}^{i} \alpha^{i} c}\left(\frac{n p}{n_{0} p_{0}}-\frac{q^{i}}{q_{0}^{i}}\right)^{-1}\right] .
$$

Another insight into the contributions of radiative recombination and its influence on the carrier lifetime is shown in Fig. 6a, b, which shows the spatial distribution of radiative and Auger process carrier lifetimes in a reverse-biased 5 - $\mu \mathrm{m}$-on- $n \mathrm{HgCdTe}$ photodiode operating at $300 \mathrm{~K}$ and $80 \mathrm{~K}$. It is clearly seen that, for a photodiode operating at $300 \mathrm{~K}$, the photon recycling effect increases the radiative carrier lifetime in the active region ( $n$-type) by orders of magnitude. In this way, the photon recycling makes Auger 1 the dominant process at room temperature rather than the classical radiative mechanism. At $80 \mathrm{~K}$, the radiative carrier lifetime seems to be about an order of magnitude higher, after considering the PR mechanism. The lifetime is limited by
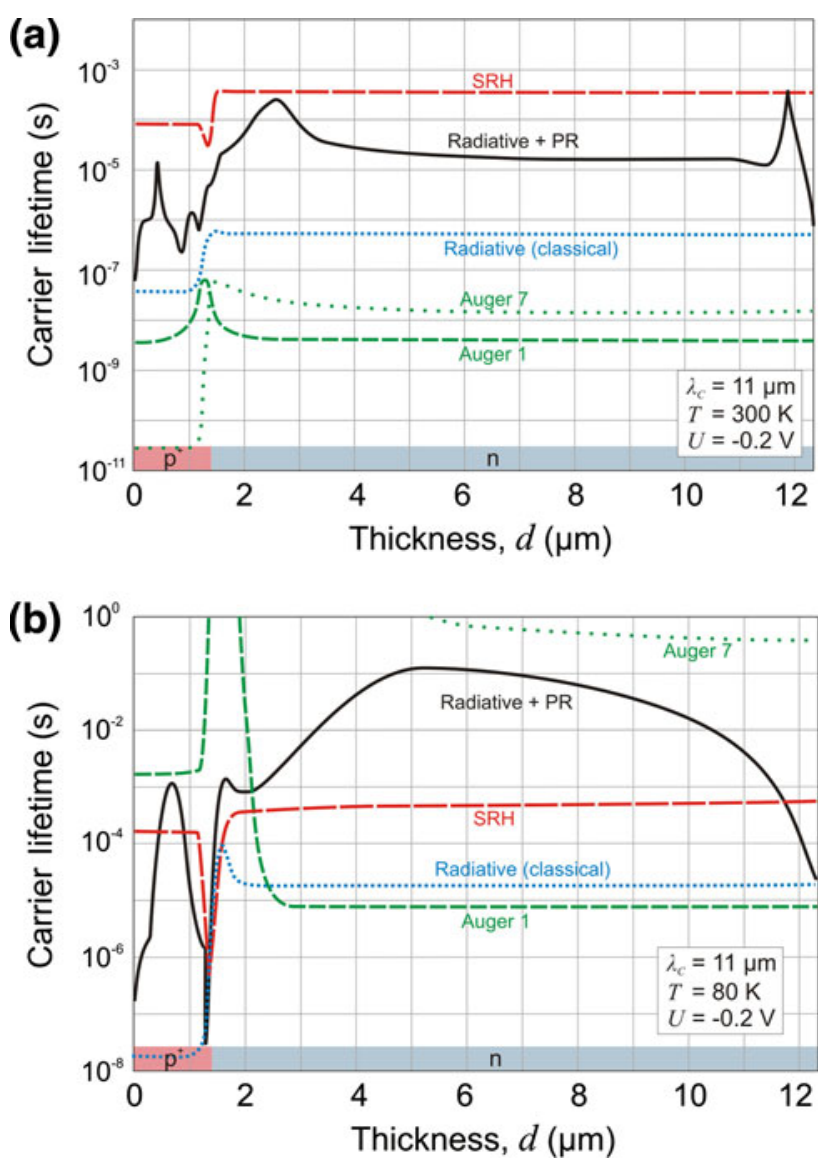

Fig. 7. Spatial distribution of carrier lifetime in a reverse-biased $11-\mu \mathrm{m} p$-on- $n \mathrm{HgCdTe}$ photodiode operating at $300 \mathrm{~K}(\mathrm{a})$ and $80 \mathrm{~K}$ (b). Reverse bias voltage is $0.2 \mathrm{~V}$.

a combination of Auger 1, PR radiative, and SRH mechanisms rather than by the classical (VRS theory) radiative mechanism.

It seems that, in long-wavelength infrared (LWIR) photodiodes, taking into account the PR effect in the radiative mechanism, Auger processes will dominate not only near room temperature but even at much lower temperatures. Similar analysis has been performed for photodiodes with cutoff wavelength of $11 \mu \mathrm{m}$. Figures $7 \mathrm{a}, \mathrm{b}$ shows the spatial distribution of radiative and Auger process carrier lifetime in a reverse-biased LWIR $p$-on- $n$ $\mathrm{HgCdTe}$ photodiode operating at $300 \mathrm{~K}$ and $80 \mathrm{~K}$.

While in the photodiode operating near room temperature Auger mechanisms prevail even over the classical (VRS theory) calculated radiative mechanism, at $80 \mathrm{~K}$ the lifetime is limited by a combination of Auger 1 and classical (VRS theory) radiative mechanisms. The $\mathrm{PR}$ effect increases the radiative carrier lifetime in the $n$-type region by two orders of magnitude, so the influence of the radiative mechanism is not essential.

Figures 8 and 9 show the spatial distribution of the net carrier radiative recombination (generation) rates in a reverse- and a forward-biased $11-\mu \mathrm{m} p$-on- $n$ $\mathrm{HgCdTe}$ photodiode operating at $300 \mathrm{~K}$ and $80 \mathrm{~K}$, 
(a)

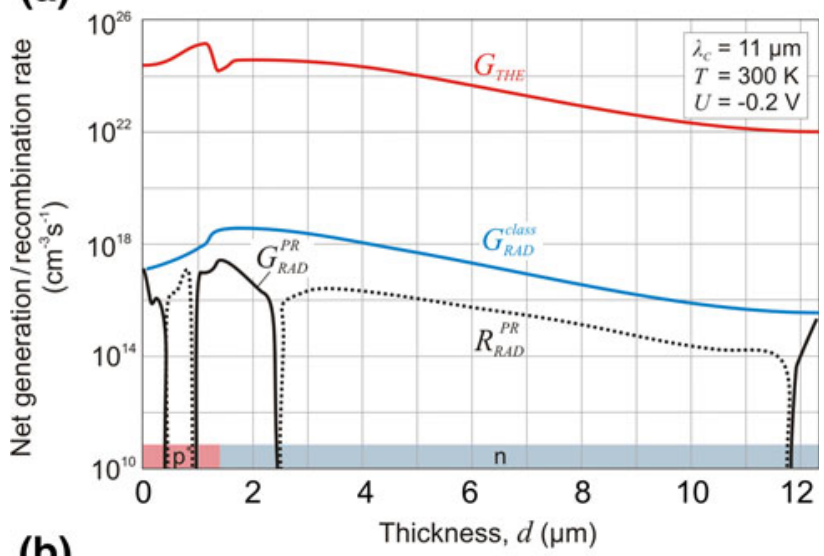

(b)

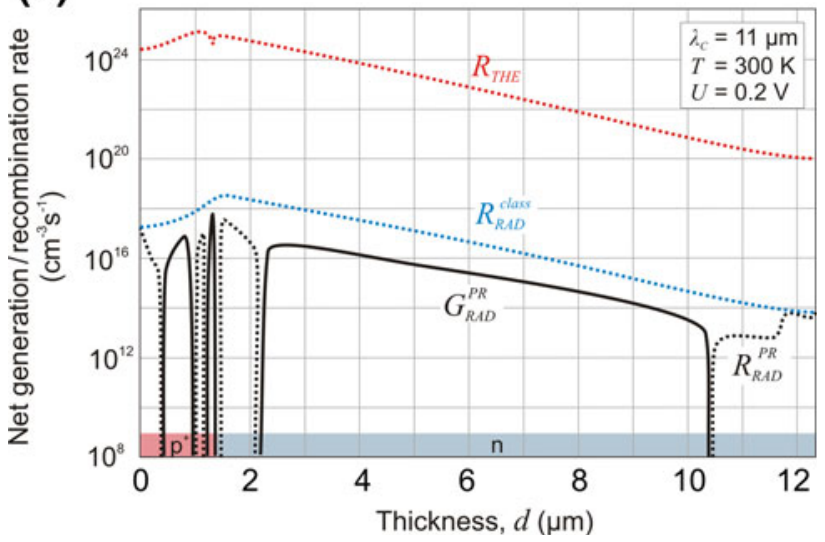

Fig. 8. The spatial distribution of net recombination (generation) rates in a reverse (a) and a forward (b) biased 11- $\mu \mathrm{m} p$-on- $n \mathrm{HgCdTe}$ photodiode operating at $300 \mathrm{~K}$. Bias voltage is $0.2 \mathrm{~V}$.

respectively. Moreover, Fig. 8 presents the net thermal generation rate, $G_{\mathrm{THE}}$, and the net thermal recombination rate, $R_{\mathrm{THE}}$, which are the results of the action of band-to-band (Auger 1 and Auger 7), $\mathrm{SRH}$, and radiative (with $\mathrm{PR}$ effect) mechanisms. It is clearly shown that the radiative mechanism does not influence the resultant thermal generation (recombination) rate in high-temperature LWIR HgCdTe photodiodes.

\section{CONCLUSIONS}

We have presented a numerical method for analyzing $\mathrm{g}-\mathrm{r}$ processes in semiconductor heterostructures including the contribution of the photon recycling effect in the interband radiative mechanism. To illustrate the method we considered MWIR and LWIR $p$-on- $n$ heterostructure HgCdTe photodiodes operating at room temperature and $80 \mathrm{~K}$. Both the distributions of carrier generation and recombination rates and the spatial photon density distribution in the photodiode structures have been obtained. It is clearly shown that the photon recycling effect drastically limits the influence of radiative recombination on the performance of $\mathrm{HgCdTe}$ photodiodes. The general conclusion once again confirms Humphreys' previous assertion that radiative
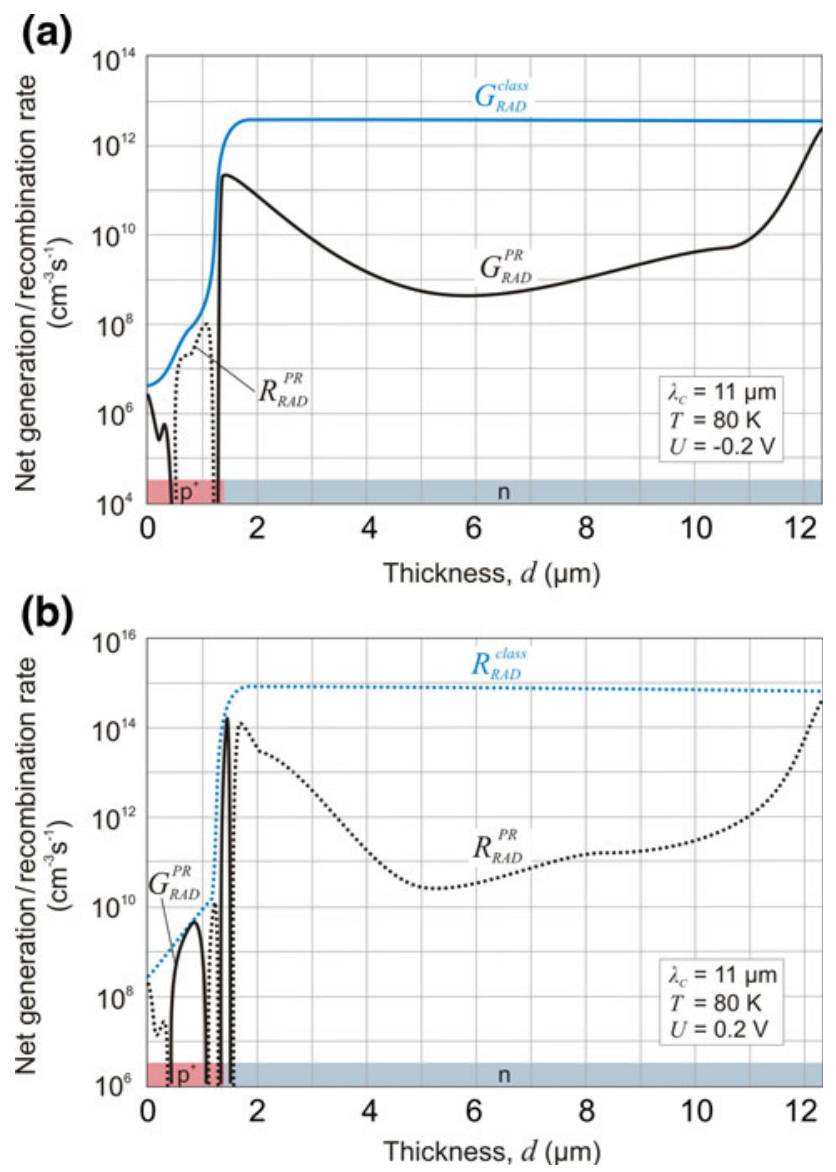

Fig. 9. The spatial distribution of net recombination (generation) rates in a reverse (a) and a forward (b) biased 11- $\mu \mathrm{m} p$-on- $n \mathrm{HgCdTe}$ photodiode operating at $80 \mathrm{~K}$. Bias voltage is $0.2 \mathrm{~V}$.

recombination, although of fundamental nature, does not limit the ultimate performance of MWIR and LWIR HgCdTe photodiodes at room temperature. Its influence can be observed at lower operating temperature.

\section{ACKNOWLEDGEMENTS}

This work was undertaken and completed with the financial support of the Polish Ministry of Science and Higher Education as Research Project No. PBZ M. Sz. W. 02/I/2007 and European Project No. POIG.01.03.01-14-016/08 "New Photonic Materials and Their Advanced Application."

\section{OPEN ACCESS}

This article is distributed under the terms of the Creative Commons Attribution License which permits any use, distribution, and reproduction in any medium, provided the original author(s) and the source are credited.

\section{REFERENCES}

1. P.V.C. Lopes, A.J. Syllaios, and M.C. Chen, Semicond. Sci. Technol. 8, 824 (1993). 
2. A. Rogalski, Infrared Detectors, 2nd ed. (Boca Raton: CRC, 2010).

3. T. Elliott, N.T. Gordon, and A.M. White, Appl. Phys. Lett. 74, 2881 (1999).

4. J. Piotrowski, Opto-Electron. Rev. 12, 111 (2004).

5. R.G. Humphreys, Infrared Phys. 23, 171 (1983).

6. W. Van Roosbroeck and W. Shockley, Phys. Rev. 94, 1558 (1954).

7. R.G. Humphreys, Infrared Phys. 26, 337 (1986).

8. D. Mihalas and B. Weibel-Mihalas, Foundation of Radiation Hydrodynamics (New York: Oxford University Press, 1984)

9. J.I. Castor, Lectures on Radiation Hydrodynamics (Cambridge: Cambridge University Press, 2004).

10. K. Jóźwikowski, M. Kopytko, and A. Rogalski, Opt. Eng. 50, 061000 (2011).
11. J.S. Blakemore, Semiconductor Statistics (Oxford: Pergamon, 1962).

12. W.W. Anderson, Infrared Phys. 20, 363 (1980).

13. S. Rolland, Properties of Narrow Gap Cadmium-based Compounds, ed. P. Capper (London: Inspec, 1994), p. 80.

14. A. Rogalski, K. Adamiec, and J. Rutkowski, Narrow-Gap Semiconductor Photodiodes (Bellingham: SPIE, 2000).

15. T. Ashley and C.T. Elliott, Electron. Lett. 21, 451 (1985).

16. T. Ashley, C.T. Elliott, N.T. Gordon, J.J. Phillips, and R.S. Hall, Infrared Phys. Technol. 38, 145 (1997).

17. C.T. Elliot, Phil. Trans. R. Soc. Lond. A 359, 567 (2001).

18. J.R. Lindle, W.W. Bewley, I. Vurgaftman, C.S. Kim, J.R. Meyer, J.L. Johnson, M.L. Thomas, E.C. Piquette, and W.E. Tennant, IEEE J. Quantum Electron. 41, 227 (2005). 